\section{Boerhaave's syndrome}

\section{Sheldon Godinho FCRad(D) SA, FRCR}

Department of Radiology, University of Pretoria

A 38-year-old woman became nauseous while driving in a car. She began retching before her husband could pull over, and attempted to swallow down. Severe chest pain ensued and gradually worsened overnight. Medical assistance was sought the following morning and the patient was referred to a local hospital for a barium swallow. An incarcerated para-oesophageal hernia was diagnosed. The patient was then referred to a general surgeon in our hospital. Noting the severity of her pain and shallow tachypnoea, we were approached for a second opinion.

The control chest radiograph showed subtle medial basal veiling on the left and a little pleural fluid (Fig. 1). An irregular outpouching could be seen on the left posterolateral aspect of the oesophagus, above the diaphragm on the swallow series

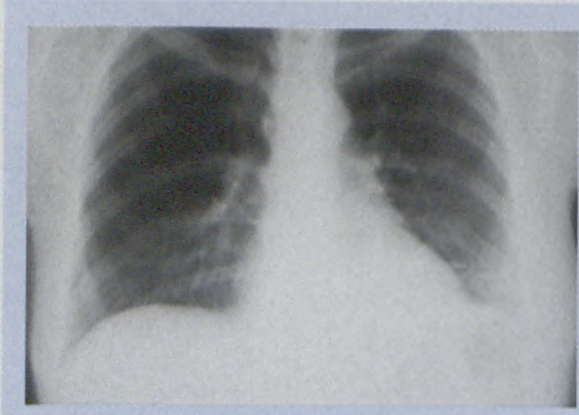

Fig. 1. Control chest before barium swallow. Note left basal infiltrate, loss of medial diaphragmatic contour and blunted costophrenic recess.
(Fig. 2). This did not appear continuous with the gastric fundus as with a para-oesophageal hernia. Repeat chest radiograph showed progression of the basal lung and pleural changes and a collection of extraluminal barium (Fig. 3). No pneumomediastinum could be seen. A presumptive diagnosis of spontaneous oesophageal rupture (Boerhaave's syndrome) was made. This was confirmed on noncontrast chest CT, which clearly showed the barium-lined cavity, perioesophageal inflammation, pleuropulmonary changes and also a small pneumomediastinum. A few pockets of upper retroperitoneal air were also visible below the hiatus (Fig. 4).

At thoractomy a $5 \mathrm{~cm}$ vertical rent was found in the left posterolateral

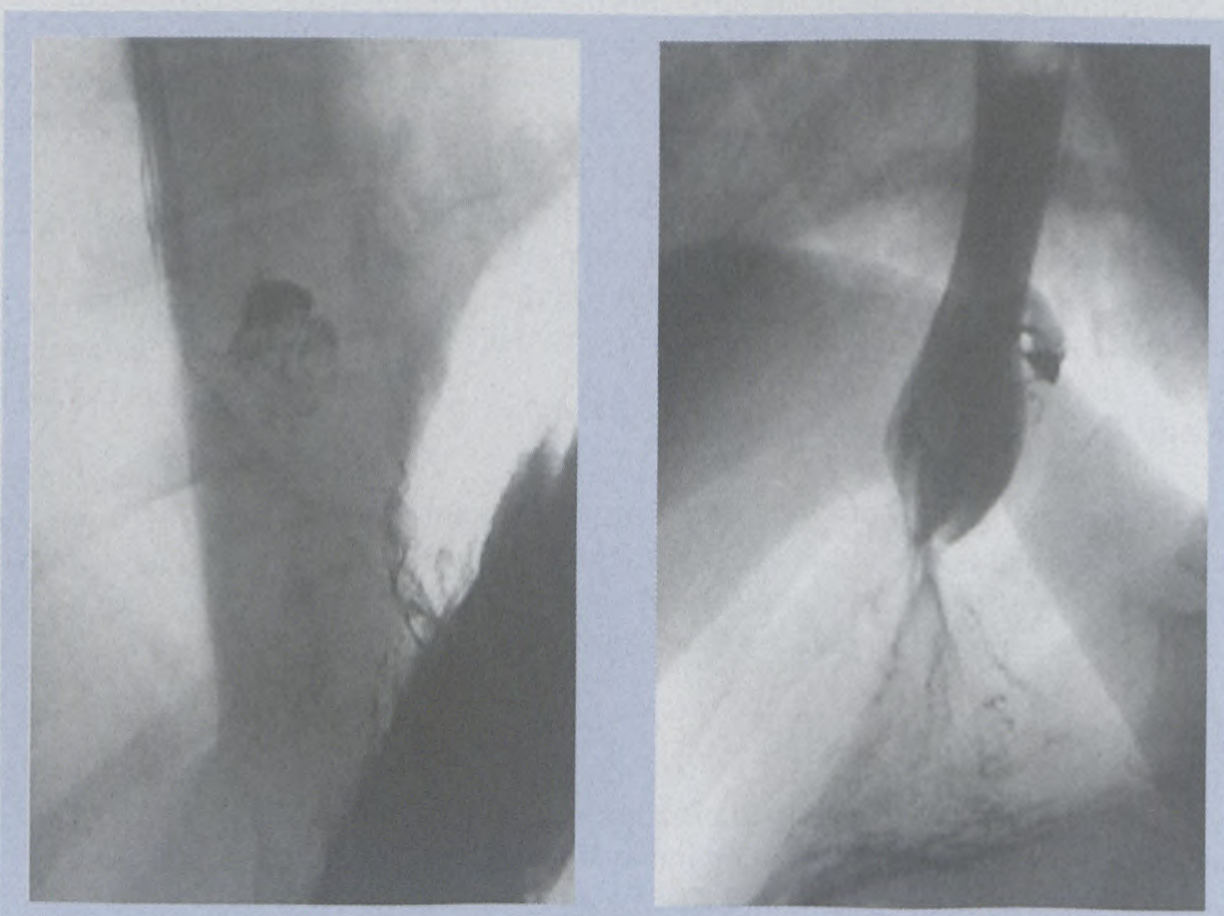

Fig. 2. Barium swallow. Extravasation of barium into a cavity located above the diaphragm, on the posterolateral aspect.

wall of the distal oesophagus, and repaired. Hospitalisation was uncomplicated and the patient was discharged 4 days later.

\section{Discussion}

Hermann Boerhaave described spontaneous rupture of the oesophagus of Lord High Admiral Wassenaer of the Royal Dutch navy in 1724, now known as Boerhaave's syndrome. This is a rare cause for oesophageal perforation, usually found in males 40 - 60 years of age secondary to forceful vomiting and often related to binge eating, drinking and alcoholism. Rare instances have been caused by straining, childbirth, severe coughing, hiccuping, seizures, asthma, and forceful swallowing. Chest pain, epigastric pain and dyspnoea are common symptoms. Hematemesis occurs in about $50 \%$ of cases and is usually not profuse. In $90 \%$ of cases a vertical tear of $1-8 \mathrm{~cm}$ occurs along the left posterolateral wall of the distal third of 


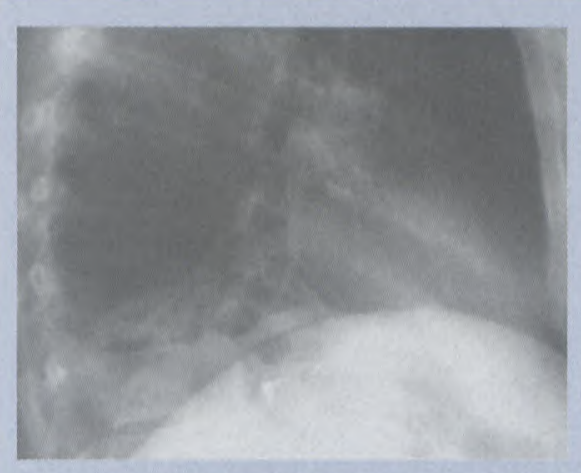

Fig. 3. Follow-up chest radiograph. Leaked barium following earlier swallow. More extensive basal veiling and pleural fluid.

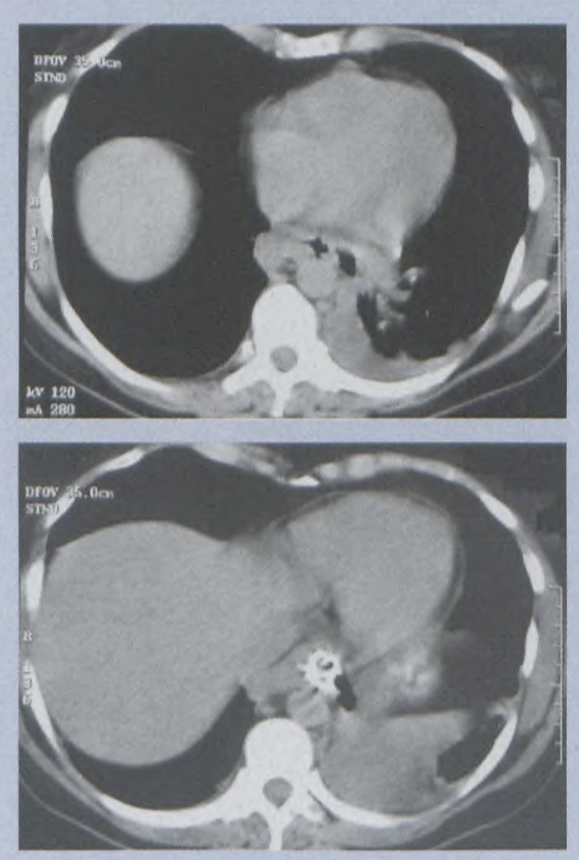

Fig. 4. CT chest. Left-sided pleural effusion and basal pulmonary infiltrate. Note mediastinal air pockets between aorta and left atrium and barium lined leakage cavity.

the oesophagus. Other clinical considerations should include a MalloryWeiss mucosal tear, aortic dissection and peptic-reflux disease.

Clinical signs are mostly nonspecific. Subcutaneous emphysema may be found in the neck or chest. Mackler's triad of vomiting, chest pain and subcutaneous emphysema is uncommon and therefore unreliable. Tachycardia, tachypnoea, fever, hypotension and shock can occur with late presentation. Rarity, unfa- miliarity and a sometimes undramatic history often delay diagnosis. Up to $50 \%$ are not diagnosed at initial presentation! Prognosis is grave: 30 - 50\% mortality can be expected despite prompt treatment and over $90 \%$ when delayed diagnosis leads to mediastinitis.

Chest X-ray findings may suggest perforation in Boerhaave's syndrome; however X-ray findings are normal in $10-15 \%$ of cases. Signs include mediastinal emphysema or frank pneumomediastinum $(60 \%)$, mediastinal widening or air fluid level, pleural effusion ( $90 \%$, usually left), pneumothorax, or hydropneumothorax. The ' $V$ ' sign of Naclerio describes a pocket of mediastinal air lodged between the aorta and diaphragm.

Gastrografin swallows are useful and must be performed in both left and right decubitus positions. Falsenegative results occur in $10-25 \%$ of cases, however. Some authorities suggest barium oesophagography if a high suspicion of oesophageal perforation exists and Gastrografin swallow is negative. In one study, $22 \%$ of cases with a high clinical suspicion and normal water-soluble contrast swallow were found to be positive for rupture when barium was used. Non-contrast CT scan offers a number of advantages, including evaluation of the pleurae, mediastinum, and aorta. The exact perforation site is usually not visible but extravasation may be seen if scanned after oral contrast, as in this case.

Complications of oesophageal rupture include mediastinitis, empyema and septic shock. Tension pyopneumothorax and vertebral osteomyelitis from a posterior perforation have been described. Patients who recover are predisposed to repeat spontaneous rupture.

The best clinical outcomes occur with primary closure within 24 hours. Prompt diagnosis is therefore critical! A nonspecific clinical picture shifts additional responsibility onto the radiologist. While mediastinal emphysema will surely call to mind this diagnosis, it only occurs in some $60 \%$ of cases. Any basal pleuropulmonary changes must therefore be viewed with great suspicion when there is a history of vomiting and also in inebriated patients unable to give a history. Aspiration would probably be the first logical diagnostic consideration but oesophageal rupture must be borne in mind. Oesophageal and/or CT should be considered where even the slightest suspicion exists.

\section{Bibliography}

1. Adamek H. Management of esophageal perforations after therapeutic upper gastrointestinal endoscopy. Scand J Gastroenterol 1997; 32: 411 414.

2. Altorjay A, Kiss J, Voros A. Nonoperative management of esophageal perforations. Is it justified? Ann Surg 1997; 225: 415-421.

3. Borotto E. Risk factors of oesophageal perforation during pneumatic dilatation for achalasia. Gut 1996; 39(1): 9-12.

4. Bufkin BL, Miller JI jun., Mansour KA. Esophageal perforation: emphasis on management. Ann Thorac Surg 1996; 61: 1447-1451; discussion 1451-1452.

5. Cordero JQ. Distal esophageal rupture after external blunt trauma: report of two cases. I Trauma 1997; 42: 321-322.

6. Inculet R, Clark C, Girvan D. Boerhaave's syn drome and children: a rare and unexpected combination. JPediatr Surg 1996; 31: 13001301.

7. Jagminas L. Boerhaave's syndrome presenting with abdominal pain and right hydropneumothorax. Am J Emerg Med 1996; 14(1): 53-56.

8. Lujan H. Recurrent spontaneous rupture of the esophagus: an unusual late complication of Boerhaave's syndrome. Surgery 1997; 122: 634636.

9. Panieri E, Millar AJ, Rode $\mathrm{H}$, et al. Iatrogenic esophageal perforation in children: patterns of injury, presentation, management, and outcome. J Pediatr Surg 1996; 31: 890-895.

10. Ring D. Vertebral osteomyelitis after blunt traumatic esophageal rupture. Spine 1995; 20(1): 98-101.

11. Sabanathan S. Surgical management of intrathoracic oesophageal rupture. Br J Surg 1994; 81: 863-865.

12. Troum S. Surviving Boerhaave's syndrome without thoracotomy. Chest 1994; 106(1):297299. 